\section{Avaliação da estimulação elétrica transcutânea do nervo para alívio de dor após cesárea: ensaio clínico randomizado}

\section{Assessment of transcutaneous electrical nerve stimulation for pain relief after cesarean section: a randomized clinical trial}

Ligia Sousa 1

Flávia Azevedo Gomes 2

Ana Carolina Rodarti Pitangui 3

Ana Márcia Spanó Nakano 4

1-4 Escola de Enfermagem de Ribeirão Preto. Universidade de São Paulo. Av. Bandeirantes, 3900. Ribeirão Preto, SP, Brasil. CEP: 14.040-902. E-mail: ligia.sousa@hotmail.com

\begin{abstract}
Objectives: to assess the use of transcutaneous electrical nerve stimulation (TENS) for post-cesarean pain relief.

Methods: a simple-blind and random clinical controlled-study was carried out. The experimental group comprised twenty puerperal women to whom the TENS technique had been applied and 20 others as a control group. The numeric rating scale and the McGill Pain Questionnare were used to evaluate pain. Three evaluations were carried out: the first before the intervention using transcutaneous electrical nerve stimulation; a second, after the intervention and a third, one hour after the intervention. For statistical analysis, the Mann-Whitney's non-parametric test was used.

Results: the experimental group presented a statistically significant larger reduction in pain than the control group on the second and the third evaluation using both the numeric rating scale $(p<0.001)$ and McGill $(p<0.001)$.

Conclusions: transcutaneous electrical nerve stimulation is effective for the reduction of pain and the resulting relief persists for an hour subsequent to its application and could be used in clinical practice as a non-pharmacological resource to promote humanization of the puerperal condition in cases of caesarean section.
\end{abstract}

Key words Cesarean section, Pain, Transcutaneous electric nerve stimulation

\section{Resumo}

Objetivos: avaliar a utilização da estimulação elétrica transcutânea do nervo no alívio de dor após cesárea.

Métodos: realizou-se um estudo clínico controlado randomizado e simples-cego. Participaram do estudo 20 puérperas no grupo experimental que recebiam a estimulação elétrica transcutânea do nervo e 20 no grupo controle. Para avaliar a dor foi usada a escala de categoria numérica e o questionário de dor McGill. Foram realizadas três avaliações: a primeira antes da intervenção com a estimulação elétrica transcutânea do nervo, a segunda após a intervenção e a terceira, uma hora após a intervenção. Para a análise estatística foi utilizado o teste nãoparamétrico de Mann-Whitney $U$.

Resultados: o grupo experimental apresentou redução maior da dor estatisticamente significante que o grupo controle na segunda e na terceira avaliação pela escala numérica de dor $(p<0,001)$ e pelo McGill $(p<0,001)$

Conclusões: a estimulação elétrica transcutânea do nervo é eficaz para a redução da dor e este alivio permanece por, pelo menos, uma hora após sua aplicação, podendo ser utilizada na prática clínica e como recurso não-farmacológico de humanização no puerpério de cesárea.

Palavras-chave Cesárea, Dor, Estimulação elétrica nervosa transcutânea 
Introdução

A cesárea deve ser utilizada quando, durante a evolução da gravidez e/ou do parto, surgem situações específicas em que o procedimento cirúrgico se torna necessário para preservar a vida da mãe e/ou do feto. ${ }^{1}$ Em países desenvolvidos, o índice de cesárea é alto quando comparado a outros países, sendo considerado um problema de saúde pública. ${ }^{2}$ $\mathrm{Na}$ América Latina este índice vem aumentando consideravelmente, quando comparado a países desenvolvidos e às taxas de cesárea indicada pela Organização Mundial da Saúde, de 10 a $15 \%$ de todos os partos. ${ }^{2}$

$\mathrm{O}$ atendimento integral à mulher no puerpério, com vistas à humanização, não deve se restringir a puérpera de parto vaginal, a cesárea também é humanizada quando sua indicação visa minimizar riscos à mãe e ao feto. A fase puerperal deste tipo de parto também deve ser humanizada, ou seja, atender às necessidades da mulher e diminuir a morbidade, tão presente no pós-operatório de cesárea. ${ }^{3}$

A cesárea está relacionada com alta taxa de morbi-mortalidade materna e perinatal. Como morbidade decorrente da cesárea, destaca-se a presença de dor no local da cirurgia. A dor pós-operatória apresenta difícil recuperação e retarda o contato da mãe com o recém-nascido, além de dificultar a amamentação, os cuidados com o recémnascido e os cuidados com a higiene pessoal. 4

Após deflagrado o movimento para humanização do ciclo gravídico-puerperal, que tem como base reduzir a medicalização excessiva e estimular o uso de estratégias que respeitem a fisiologia dos processos, observa-se incentivo à pesquisa e utilização de métodos não-farmacológicos para alívio da dor durante trabalho de parto e puerpério, seja parto normal ou cesárea. ${ }^{3}$

A estimulação elétrica transcutânea do nervo (TENS) é uma alternativa não-invasiva, simples, de baixo investimento e de fácil aplicação, utilizada para reduzir a dor aguda, como dor após cesárea. A TENS promove alívio imediato da dor e não apresenta efeitos colaterais indesejáveis. 5,6

Alguns autores 7 sugerem que a estimulação elétrica transcutânea do nervo é um recurso terapêutico seguro, pois trata-se de uma corrente despolarizada, ou seja, a corrente elétrica gerada não causa queimaduras. Os estudos que utilizaram este recurso após a cesárea mostraram sua efetividade no menor consumo de medicamentos farmacológicos, maior alívio de dor, menor tempo de internação hospitalar, mobilização precoce e melhor padrão respiratório.7-10
Baseado nisto, o presente estudo tem como objetivo avaliar a efetividade da estimulação elétrica transcutânea do nervo no alívio de dor da ferida operatória após cesárea.

\section{Métodos}

Esta pesquisa caracteriza-se por um ensaio clínico randomizado, controlado e simples-cego. O estudo foi aprovado pelo Comitê de Ética em Pesquisa da Escola de Enfermagem de Ribeirão Preto da Universidade de São Paulo, sob o protocolo número 0660/2006. O estudo foi realizado na Maternidade do Complexo Aeroporto (MATER), situada em Ribeirão Preto, no Estado de São Paulo, Brasil, nos meses de março, abril e maio de 2007.

Como critérios de inclusão da amostra, apresentam-se idade superior a 18 anos, não obesas, raquianestesia antes do procedimento cirúrgico (dosagem das drogas semelhantes para todas as puérperas), incisão tipo Pfannestiel, primiparidade ou multiparidade, alfabetizadas e com capacidade de compreensão das escalas de dor utilizadas, ter realizado a cesárea entre 7 horas e 18 horas. As seguintes indicações de cesárea foram incluídas: iteratividade, falha de progressão do trabalho de parto, sofrimento fetal agudo, apresentação pélvica fetal e desproporção céfalo pélvica. Foi estabelecido que, as participantes que apresentassem qualquer intercorrência após a cesárea, tais como hemorragia ou infecção, seriam excluídas da pesquisa.

O cálculo do tamanho amostral foi realizado no programa PASS, pela fórmula de diferença de médias após análise de um estudo piloto. Utilizou-se como parâmetro a redução nos escores de dor após a intervenção de dois pontos na escala de categoria numérica como clinicamente relevante para alívio de dor com um desvio padrão de 1,8 e 1,0 para o grupo de intervenção e o controle, respectivamente. Obteve-se uma amostra de 36 sujeitos para um poder de $90 \%$ e um nível de confiança de 99\%. Após determinado o tamanho da amostra, optou-se em analisar 20 sujeitos para cada grupo de estudo, visando aumentar o poder estatístico da amostra.

Das 53 puérperas elegíveis para o estudo, cinco referiram ausência de dor e sete delas eram obesas, não atendendo aos critérios de inclusão da pesquisa. Apenas uma puérpera que atendia aos critérios de inclusão recusou-se em participar da pesquisa, restando 40 participantes, que foram randomizadas em dois grupos pelo site www.randomization.com.

As 20 puérperas que pertenciam ao grupo experimental foram submetidas aos procedimentos de 
rotina da instituição e ao tratamento com TENS convencional próxima à incisão cirúrgica. No grupo controle, as puérperas foram submetidas aos procedimentos de rotina da instituição, sendo acompanhadas durante o período da pesquisa.

Para caracterização da amostra, as puérperas foram questionadas quanto às variáveis sóciodemográficas e obstétricas. Para avaliar a dor foi utilizada a escala de categoria numérica, cuja extremidade esquerda, ou grau 0 de dor, significa ausência de dor e aumenta gradativamente até extremidade direita, ou grau 10 de dor, que representa nível de dor extremo. ${ }^{11}$ Foram dadas instruções quanto ao uso e aplicação da escala à puérpera, conceituando a escala por meio de descritores verbais $(0=$ sem dor; $1-3=$ dor branda; $4-6=$ dor moderada; $7-10=$ dor severa). Em seguida a puérpera foi questionada da seguinte maneira em relação à Escala Numérica: "Qual número você dá para a dor que está sentindo agora?"

O Questionário de Dor McGill, adaptado e validado para a língua portuguesa por Castro12 em 1999, foi utilizado para avaliar a dor quantitativa e qualitativamente. O McGill é formado por um conjunto de 68 palavras divididas em 20 categorias que descrevem experiências dolorosas em geral. As puérperas participantes do estudo foram orientadas a escolher apenas uma ou nenhuma palavra de cada categoria. Essas palavras eram lidas às puérperas e, caso não compreendessem seu significado, este era explicado. Por meio do McGill foi possível obter o índice de avaliação da dor, baseado na soma dos valores numéricos associados às palavras escolhidas; o número de palavras escolhidas pelas puérperas e a intensidade de dor presente por meio de palavras âncora: (0) sem dor, (1) fraca, (2) moderada, (3) forte, (4) violenta e (5) insuportável.

Em razão da dificuldade de realizar estudos placebos ou cegos com a TENS, pela presença do aparelho e estímulos produzidos pela corrente, 13 optou-se em utilizar dois pesquisadores, um principal e um auxiliar. O pesquisador principal era responsável em realizar as avaliações dos grupos estudados, sem saber a qual grupo cada mulher pertencia, e o pesquisador auxiliar aplicava a TENS no grupo experimental ou apenas acompanhava as puérperas do grupo controle. Isto garante o encobrimento do estudo e aumenta o rigor metodológico da pesquisa, pois evita interferência do pesquisador na resposta dos sujeitos. ${ }^{14}$

O estudo foi iniciado 24 horas após a cesárea. Para aquelas participantes do grupo experimental, era aplicada a TENS por meio da técnica bipolar cruzada, um centímetro acima e abaixo da incisão cirúrgica, utilizando quatro eletrodos de cinco por três centímetros de borracha siliconada e carbonada com gel condutor de $\mathrm{pH}$ neutro e fita adesiva hipoalérgica para fixá-los. ${ }^{9}$ Foi utilizado aparelho portátil de estimulação elétrica transcutânea do nervo KW Compact, programado para gerar uma corrente com largura de pulso de 75 microssegundos e frequência de $100 \mathrm{Hertz}$. O controle de intensidade foi feito pela própria puérpera e ajustado sempre que necessário para que ela sentisse uma sensação de formigamento forte e bem tolerada. $15 \mathrm{O}$ aparelho permaneceu ligado por 45 minutos. As puérperas do grupo controle foram acompanhadas pelo pesquisador auxiliar pelo mesmo tempo. Nos dois grupos de estudo, as puérperas permaneceram deitadas durante os 45 minutos de intervenção, para padronização do estudo.

A dor foi avaliada com a puérpera em repouso, três vezes: ao iniciar o estudo (primeira avaliação); após os 45 minutos de tratamento para o grupo experimental e acompanhamento para o controle (segunda avaliação) e uma hora após a segunda avaliação (terceira avaliação). Durante o período do estudo as participantes permaneceram sem medicamento, porém, caso achassem necessário poderiam fazer uso dele, sendo excluídas da pesquisa.

Para a análise dos dados, utilizou-se o programa Statical Package for Social Scienses (SPSS, versão 11.5). Foi realizada uma análise descritiva dos dados para caracterização sócio-demográfica das participantes do estudo. O teste qui-quadrado foi utilizado para verificar se havia diferença estatística entre as participantes dos grupos estudados. Para realizar a análise comparativa dos dados referentes às escalas de categoria numérica, índice de avaliação da dor, número de palavras escolhidas e intensidade de dor presente utilizou-se o teste de Mann-Whitney U, teste não-paramétrico usado para análise comparativa de amostras pequenas que não apresentam distribuição normal. O teste qui-quadrado foi utilizado para verificar se houve associação entre a paridade e a intensidade de dor sentida na primeira avaliação.

\section{Resultados}

Nenhuma participante foi excluída durante a coleta por solicitação de medicamento. Não foi observada nenhuma alteração cutânea como irritação ou hiperemia no grupo que utilizou a TENS.

A amostra constou de 40 puérperas no pós-operatório de cesárea, com idades variando de 18 a 38 anos no grupo experimental (mediana de 27,5 anos); 
Tabela 1

Frequência e percentual das variáveis sócio-demográficas dos grupos estudados. Ribeirão Preto, São Paulo, 2007.

\begin{tabular}{|c|c|c|c|c|c|c|}
\hline \multirow[t]{2}{*}{ Variáveis sócio-demográficas } & \multicolumn{2}{|c|}{ Experimental } & \multicolumn{2}{|c|}{ Controle } & \multirow[t]{2}{*}{ Total } & \multirow[t]{2}{*}{$p^{*}$} \\
\hline & $\mathrm{n}$ & $\%$ & $\mathrm{n}$ & $\%$ & & \\
\hline \multicolumn{7}{|l|}{ Faixa etária } \\
\hline $18 \mid-25$ & 7 & 35,0 & 13 & 65,0 & 20 & \\
\hline $26 \mid-35$ & 11 & 55,0 & 4 & 15,0 & 15 & 0,262 \\
\hline $36 I-45$ & 2 & 10,0 & 3 & 20,0 & 5 & \\
\hline \multicolumn{7}{|l|}{ Ocupação } \\
\hline Atividade não remunerada & 14 & 70,0 & 14 & 70,0 & 28 & 1 \\
\hline Atividade remunerada & 6 & 30,0 & 6 & 30,0 & 12 & \\
\hline \multicolumn{7}{|l|}{ Estado marital } \\
\hline Não vive com companheiro & 2 & 10,0 & 1 & 5,0 & 3 & 0,553 \\
\hline Vive com companheiro & 18 & 90,0 & 19 & 95,0 & 37 & \\
\hline \multicolumn{7}{|l|}{ Vício } \\
\hline Sim & 2 & 10,0 & 4 & 20,0 & 6 & 0,637 \\
\hline Não & 18 & 90,0 & 16 & 80,0 & 34 & \\
\hline \multicolumn{7}{|l|}{ Escolaridade } \\
\hline Alfabetizada & 20 & 100,0 & 20 & 100,0 & 40 & 1 \\
\hline Não alfabetizada & - & - & - & - & - & \\
\hline \multicolumn{7}{|l|}{ Índice de Massa Corporal } \\
\hline Baixo Peso & 1 & 5,0 & 1 & 5,0 & 2 & \\
\hline Adequado & 13 & 65,0 & 7 & 35,0 & 20 & 0,108 \\
\hline Sobrepeso & 6 & 30,0 & 12 & 60,0 & 18 & \\
\hline
\end{tabular}

*Teste de qui-quadrado

Tabela 2

Frequência e percentual das puérperas dos grupos estudados, segundo as variáveis obstétricas, Ribeirão Preto, São Paulo, 2007.

\begin{tabular}{|c|c|c|c|c|c|c|}
\hline \multirow[t]{2}{*}{ Variáveis obstétricas } & \multicolumn{2}{|c|}{ Experimental } & \multicolumn{2}{|c|}{ Controle } & \multirow[t]{2}{*}{ Total } & \multirow[t]{2}{*}{$p^{*}$} \\
\hline & $\mathrm{n}$ & $\%$ & $\mathrm{n}$ & $\%$ & & \\
\hline \multicolumn{7}{|l|}{ Idade gestacional } \\
\hline Pré-termo & 2 & 10,0 & 1 & 5,0 & 3 & 0,553 \\
\hline A termo & 18 & 90,0 & 19 & 95,0 & 37 & \\
\hline \multicolumn{7}{|l|}{ Abortos anteriores } \\
\hline Nenhum & 18 & 90,0 & 13 & 65,0 & 31 & 0,062 \\
\hline Um ou dois abortos anteriores & 2 & 10,0 & 7 & 35,0 & 9 & \\
\hline \multicolumn{7}{|l|}{ Paridade } \\
\hline Primípara & 7 & 35,0 & 9 & 45,0 & 16 & 0,626 \\
\hline Multípara & 13 & 65,0 & 11 & 55,0 & 24 & \\
\hline \multicolumn{7}{|l|}{ Consulta pré-natal } \\
\hline$\leq 5$ consultas & 3 & 15,0 & 2 & 10,0 & 5 & 0,637 \\
\hline$\geq 6$ consultas & 17 & 85,0 & 18 & 90,0 & 25 & \\
\hline \multicolumn{7}{|l|}{ Cesárea atual } \\
\hline Eletiva & 4 & 20,0 & 6 & 30,0 & 10 & 0,471 \\
\hline Não eletiva & 16 & 80,0 & 14 & 70,0 & 30 & \\
\hline \multicolumn{7}{|l|}{ Esterilizarão tubárea } \\
\hline $\operatorname{sim}$ & 2 & 10,0 & - & - & 2 & 0,152 \\
\hline Não & 18 & 90,0 & 20 & 100,0 & 38 & \\
\hline
\end{tabular}

*Teste de qui-quadrado 
Diferença da intensidade da dor presente, índice de avaliação da dor, número de palavras escolhidas e escala de categoria numérica entre a primeira e segunda avaliação. Ribeirão Preto, São Paulo, 2008.

\begin{tabular}{|c|c|c|c|c|c|c|c|}
\hline \multirow[t]{2}{*}{ Variável } & \multicolumn{3}{|c|}{ Experimental } & \multicolumn{3}{|c|}{ Controle } & \multirow[t]{2}{*}{$p^{*}$} \\
\hline & $\begin{array}{c}\text { Primeira } \\
\text { (mediana) }\end{array}$ & $\begin{array}{l}\text { Segunda } \\
\text { (mediana) }\end{array}$ & $\begin{array}{c}\text { Diferença da } \\
\text { mediana }\end{array}$ & $\begin{array}{l}\text { Primeira } \\
\text { (mediana) }\end{array}$ & $\begin{array}{l}\text { Segunda } \\
\text { (mediana) }\end{array}$ & $\begin{array}{l}\text { Diferença da } \\
\text { mediana }\end{array}$ & \\
\hline Intensidade da dor presente & 2,50 & 2,00 & 0,50 & 2,00 & 2,00 & 0,00 & 0,001 \\
\hline Índice de avaliação da dor & 22,50 & 8,50 & 14,00 & 17,50 & 16,00 & 1,50 & $<0,001$ \\
\hline Número de palavras escolhidas & 11,50 & 5,00 & 6,50 & 8,00 & 8,00 & 0,00 & $<0,001$ \\
\hline Escala de categoria numérica & 5,00 & 0,00 & 5,00 & 5,00 & 5,00 & 0,00 & $<0,001$ \\
\hline
\end{tabular}

*Teste de Mann-Whitney U.

\section{Tabela 4}

Diferença da intensidade da dor presente e escala de categoria numérica entre a primeira e terceira avaliação. Ribeirão Preto, São Paulo, 2008.

\begin{tabular}{|c|c|c|c|c|c|c|c|}
\hline \multirow[t]{2}{*}{ Variável } & \multicolumn{3}{|c|}{ Experimental } & \multicolumn{3}{|c|}{ Controle } & \multirow[t]{2}{*}{$p^{*}$} \\
\hline & $\begin{array}{l}\text { Primeira } \\
\text { (mediana) }\end{array}$ & $\begin{array}{l}\text { Segunda } \\
\text { (mediana) }\end{array}$ & $\begin{array}{c}\text { Diferença da } \\
\text { mediana }\end{array}$ & $\begin{array}{l}\text { Primeira } \\
\text { (mediana) }\end{array}$ & $\begin{array}{l}\text { Segunda } \\
\text { (mediana) }\end{array}$ & $\begin{array}{c}\text { Diferença da } \\
\text { mediana }\end{array}$ & \\
\hline Intensidade da dor presente & 2,50 & 1,00 & 1,50 & 2,00 & 1,50 & 0,50 & 0,006 \\
\hline Escala de categoria numérica & 5,00 & 4,00 & 1,00 & 5,00 & 2,00 & 3,00 & $<0,001$ \\
\hline
\end{tabular}

*Teste de Mann-Whitney U.

no grupo controle, a idade ficou entre 19 a 44 anos (mediana de 24 anos). Não houve diferença estatística significativa na média de idade entre os grupos estudados $(p=0,262)$. A paridade apresentou mediana de 1,0 no grupo experimental e no grupo controle. As Tabelas 1 e 2 mostram as frequências e porcentagens das principais variáveis sócio-demográficas e obstétricas encontradas nos grupos de estudo. Em relação à indicação para a cesárea, sete puérperas do grupo experimental e cinco do grupo controle tiveram cesárea por iteratividade. A desproporção céfalo-pélvica ou apresentação pélvica foi presente em duas do grupo experimental e duas do controle; sofrimento fetal agudo em quatro do experimental e seis do grupo controle e outras indicações relacionadas à falha de progressão do trabalho de parto foram indicação para cesárea em sete puérperas do grupo experimental e sete do grupo controle. Por se tratar de estudo randomizado, a aleatorização da amostra garantiu homogeneidade das variáveis sócio-demográficas e obstétricas, os grupos estudados não apresentaram diferenças estatísticas significativas.

$\mathrm{Na}$ abordagem qualitativa do McGill os descritores relacionados à dor mais citados pelas puérperas dos grupos estudados foram: que prende $(62,5 \%)$, que repuxa $(60 \%)$, câimbra/cólica $(57,5 \%)$, dolorido $(50 \%)$, chata $(45 \%)$, que cansa $(37,5 \%)$, pontada de faca $(35 \%)$ e latejante $(30 \%)$.

Os grupos estudados não apresentaram distribuição normal de suas variáveis ordinais contínuas na intensidade de dor presente $(p=0,010)$, índice de avaliação da dor $(p=0,029)$ e número de palavras escolhidas $(p=0,025)$ do questionário de dor McGill na primeira avaliação.

A intensidade de dor presente apresentou, na avaliação inicial, mediana de 2,50 para o grupo experimental e de 2,00 para o grupo controle. Foi encontrada uma diferença estatisticamente significativa da intensidade da dor presente entre a primeira e a segunda avaliação e entre a primeira e a terceira avaliação nos grupos estudados, mostrando que o uso da TENS apresenta uma diferença no alívio da dor significativamente maior que o não uso (Tabelas 3 e 4$)$.

Na primeira avaliação, o índice de avaliação da dor do questionário de dor McGill apresentou mediana de 22,50 para o grupo experimental e de 
17,50 para o grupo controle. O número de palavras escolhidas apresentou mediana de 11,50 para o grupo experimental e mediana de 8,00 para o grupo controle. Por meio do índice de avaliação da dor e do número de palavras escolhidas observou-se uma maior redução entre a primeira e a segunda avaliação estatisticamente significativa da dor no grupo experimental quando comparado ao controle (Tabela 3).

A escala de categoria numérica, na primeira avaliação, apresentou mediana de 5,00 para o grupo experimental e para o grupo controle. Os valores obtidos nos grupos estudados na primeira avaliação não apresentaram diferença estatística significativa $(p=0,857)$. Na análise comparativa da escala de categoria numérica em relação aos grupos de estudo, encontrou-se diferença significativa no alívio de dor entre os dois grupos de estudos da primeira para a segunda avaliação de dor (Tabelas 3 e 4). O mesmo foi observado no alívio de dor entre a primeira e terceira avaliação. Encontrou-se uma diferença estatisticamente significante entre os grupos de estudo, sugerindo que houve uma diminuição estatística significativa da dor para aquelas puérperas que utilizaram a TENS, quando comparada àquelas que não a utilizaram por pelo menos uma hora após a intervenção no grupo experimental e acompanhamento no grupo controle (Tabela 4).

Foi realizado o teste qui-quadrado para verificar se houve associação entre escores de dor da escala de categoria numérica e o nível de paridade. As primíparas e as multíparas apresentaram mediana de 5,00 , não apresentando associação estatística significativa com o nível de dor.

\section{Discussão}

Este estudo avaliou a eficácia da Estimulação Elétrica Transcutânea do Nervo no alívio da dor após cesárea. Os resultados obtidos mostraram que a TENS promove alívio de dor no grupo que a utilizou. Os resultados apresentados são capazes de sugerir a efetividade da TENS para reduzir dor após cesárea imediatamente após seu uso.

Por se tratar de estudo randomizado, não foram observadas diferenças sócio-demográficas e obstétricas significativas entre os grupos estudados, que poderiam gerar vícios nos resultados, garantindo assim a homogeneidade da amostra do estudo.

Neste estudo foi possível observar as qualidades de dor mais frequentemente relatadas pelas puérperas nas quatro categorias do McGill: sensorial, afetiva, subjetiva e mista, sendo os descritores escolhidos semelhantes entre os grupos estudados. Smith et al. ${ }^{17}$ utilizaram o McGill para avaliar a dor após cesárea, no entanto não foram relatados quais os descritores mais citados. Estudos relatam apenas que dor visceral (cólica) é frequentemente citada pelas puérperas, 7,14 sendo o mesmo observado no presente estudo, em que o descritor cólica é citado por 57,5\% das puérperas dos grupos estudados.

Os escores da intensidade de dor presente foram analisados na primeira, segunda e terceira avaliação. Apfelbaum et al.16 encontraram em pesquisa com pacientes pós-cirúrgicos que $80 \%$ deles relatam dor pós-operatória de intensidade moderada a severa. Em consonância, $40 \%$ e $30 \%$ das puérperas do grupo experimental tiveram dor inicial moderada e forte, respectivamente; no grupo controle $70 \%$ relataram dor moderada e $10 \%$ dor forte. Smith et al. 17 utilizaram os índices da intensidade de dor presente para avaliar a redução da dor após uso de TENS em mulheres submetidas à cesárea e encontraram significante redução da dor em dois dias de tratamento com a TENS. O presente estudo observou redução significativa da dor pela intensidade de dor presente, logo após e uma hora após o tratamento (segunda e terceira avaliação) no grupo experimental quando comparado ao grupo controle.

O índice de avaliação da dor do McGill mostrou significante diferença na redução de dor após cesárea com uso de TENS, sendo que o grupo experimental teve maior redução quando comparado ao controle. Smith et al. 17 utilizaram o índice de avaliação da dor para avaliar a dor após cesárea e encontraram significativa redução da dor durante três dias de tratamento com TENS quando comparado a um grupo placebo.

A redução de dor após a intervenção terapêutica foi avaliada pelo número de palavras escolhidas do questionário de dor McGill. No grupo experimental houve redução de 6,5 palavras, enquanto no grupo controle não houve redução das palavras. Isso mostra uma redução estatisticamente significativa da dor no grupo experimental. Em busca na literatura, não foi encontrado, até o momento, outro estudo que avaliou a TENS para alívio de dor após cesárea por meio do número de palavras escolhidas do McGill.

Por meio da Escala de Categoria Numérica, encontrou-se média de dor de 4,75 para o grupo experimental e 4,65 para o grupo controle. Escores de dor mais elevados foram observados por Melo de Paula et al., ${ }^{9}$ que encontraram média de dor de 7 antes do início do tratamento para o grupo experimental e 6,8 para o grupo placebo. Estes escores mostram que a dor é bastante presente após a cesárea.

Por meio da escala numérica, observou-se 
redução da dor no grupo experimental quando comparado ao grupo controle da primeira para a segunda avaliação. No presente estudo e nos estudos desenvolvidos por Melo de Paula et al., ${ }^{9}$ Rakel e Frantz, ${ }^{10}$ Lantsev e Smirnov 18 observou-se redução significativa nos escores da dor avaliados por meio de escala numérica quando comparados ao grupo controle ou ao grupo placebo. Wang et al. ${ }^{19}$ notaram maior redução nos escores de dor avaliados pela Escala Visual Analógica após terapia com TENS de alta frequência quando comparada a baixa frequência ou placebo. Uma hora após a aplicação de TENS no grupo experimental e o acompanhamento do grupo controle, as mulheres foram novamente avaliadas para verificar o efeito residual da TENS. Notou-se que, após este período, as puérperas do grupo experimental permaneciam sem dor. Wang et al.19 inferem que o efeito residual da TENS é de aproximadamente 45 minutos, e Rakel e Frantz10 fizeram nova aplicação após três horas, período em que a dor provavelmente volta ao seu valor basal. De acordo com a literatura, é controverso o período de efeito da TENS, sendo necessários mais estudos que avaliem e comprovem estes dados.

Vários estudos $8,17,20$ abordam o uso da TENS no pós-operatório e avaliam a redução da dor por meio da diminuição no consumo de analgésico entre grupos estudados, sendo que a maioria observa redução da dor através desta técnica de avaliação. Apenas um estudo encontrado não observou alívio da dor através da avaliação de consumo de medicamento, no entanto os autores atribuem seus resultados a falhas metodológicas. 21

Por motivos práticos de coleta e rotina da maternidade onde se realizou a pesquisa, a TENS foi aplicada uma única vez em cada participante do grupo experimental, que foram avaliadas uma hora após a aplicação, apresentando curto período de coleta de dados, assim como Melo de Paula. ${ }^{9}$ Smith et al. ${ }^{17}$ utilizaram a TENS durante três dias consecutivos nas puérperas e Nuñez e Carrasco ${ }^{7}$ avaliaram a TENS durante quatro horas; em ambos estudos observou-se redução no consumo analgésico e alívio da dor.

Nos dois grupos de estudo a maioria das puérperas eram multíparas, porém não houve diferença de primíparas e multíparas entre os grupos estudados, devido à randomização. Smith et al.17 relataram em seu estudo que mulheres primíparas têm experiência de dor pós-parto consideravelmente diferente de multíparas, justificando sua amostra constituída apenas de multíparas. Já Melo de Paula et al. 9 avaliaram primíparas ou mulheres no segundo parto, buscando evitar vieses de amostra. Para Niven e Murphy-Black,22 que avaliaram dor durante trabalho de parto, a dor tende a ser minimizada e melhor suportada por mulheres que tiveram mais de dois partos. No entanto, não foram encontrados estudos que descrevessem a diferença de dor presente após parto cesárea entre primíparas e multíparas.

Neste estudo, a aplicação da TENS iniciou-se 24 horas após a cesárea. Wilder-Smith et al. ${ }^{23}$ citam o segundo dia após cesárea como o maior nível de dor referido. Após esse tempo já não existem mais os efeitos de qualquer anestesia, não afetando mais os escores de dor e efeitos da TENS. 17 Além disso, é a fase em que os movimentos das puérperas começam a ser exacerbados para o auto-cuidado e cuidados com o recém-nascido, podendo desencadear aumento da dor. 23 Outros estudos 7,17,20 iniciaram a aplicação de TENS em diferentes momentos, como imediatamente após a cesárea, assim que a puérpera chegava à sala de recuperação, e também obtiveram resultados positivos quanto ao efeito da TENS.

No presente estudo, optou-se por utilizar dois pares de eletrodos colocados acima e abaixo da incisão cirúrgica por meio da técnica bipolar cruzada, que permite uma concentração da percepção da corrente na região dolorosa. Essa técnica foi utilizada anteriormente no estudo de Melo de Paula et al., 9 obtendo resultados positivos no alívio de dor e foi a técnica preferida pelas puérperas avaliadas em teste piloto. Rakel e Frantz10 e Nuñez e Carrasco ${ }^{7}$ acoplaram os eletrodos paralelamente ao lado da incisão cirúrgica, pois, segundo estes autores, a dor da incisão cirúrgica provém principalmente do trauma tecidual e muscular como resultado da retração cirúrgica, mais que da própria incisão. Essa mesma técnica foi usada por Reynolds et al. ${ }^{21}$ e não se mostrou eficaz no tratamento da dor pela TENS. Outras formas de aplicação dos eletrodos, como um par de eletrodos colocados acima e abaixo da incisão, 17 mostraram-se eficazes para o alívio de dor.

Os parâmetros de estimulação são fatores determinantes no sucesso da terapia com TENS. A frequência, a intensidade e o tempo de tratamento podem causar divergentes resultados em estudos com TENS. Assim como o presente estudo, outros utilizam a TENS convencional, ou seja, alta frequência, baixa amplitude de estimulação e intensidade forte e tolerável ajustada pelo paciente, encontrando efetividade no tratamento. ${ }^{18,19}$ Para Tsen et al.,24 a alta frequência, utilizando-se corrente de 50 a $100 \mathrm{~Hz}$, apresenta maior eficácia na inibição nociceptiva. No entanto, entre os estudos existem diferentes parâmetros, tempo de tratamento e colo- 
cação dos eletrodos, dificultando as comparações entre os tratamentos e definição do melhor parâmetro de estimulação.

A corrente da TENS ou o uso do gel e da fita adesiva podem, em alguns casos, causar irritações de pele, no entanto, entre as puérperas participantes do grupo experimental, não foi observada qualquer irritação de pele após a aplicação da TENS, assim como sugerem alguns autores.5,6 A maioria dos estudos que avaliam a TENS não descrevem o surgimento de alterações cutâneas após a aplicação da terapêutica. ${ }^{8}$

Os resultados desta investigação possibilitaram identificar que a utilização da TENS convencional reduz a dor no pós-operatório de cesárea. O presente estudo revelou que a TENS convencional pode ser utilizada em instituições de saúde que realizam partos, por ser uma técnica segura, de baixo custo e sem efeitos colaterais para a puérpera; que pode integrar como recurso não-farmacológico de alívio de

\section{Referências}

1. Silva SALC, Moraes Filho OB, Costa CFF. Análise dos fatores de risco anteparto para ocorrência de cesárea. Rev Bras Ginecol Obstet. 2005; 27: 189-96.

2. WHO (World Health Organization). Appropriate technology for birth. Lancet. 1985; 2: 436-7.

3. Brasil. Ministério da Saúde. Secretaria de políticas de Saúde. Área técnica de Saúde da Mulher. Parto, aborto e puerpério: assistência humanizada à mulher. Brasília, DF; 2003.

4. Granot M, Lowenstein L, Yarnitsky D, Tamir A, Zimmer EZ. Postcesarean section pain prediction by preoperative experimental pain assessment. Anesthesiology. 2003; 98: 1422-6.

5. Sluka KA, Walsh D. Transcutaneous electrical nerve stimulation: basic science mechanisms and clinical effectiveness. J Pain. 2003; 4: 109-21.

6. Walsh DM, Howe TE, Johnson MI, Sluka KA. Transcutaneous electrical nerve stimulation for acute pain (Protocol for a Cochrane Review). In: The Cochrane library; 2007.

7. Nuñez CN, Carrasco MFP. Estimulatión eléctrica transcutánea (EET) para reducir el dolor después de la cesárea. Ginecol Obstetr Mex. 2000; 68: 60-3.

8. Bjordal JM, Johnson MI, Ljunggreen AE. Transcutaneous electrical nerve stimulation (TENS) can reduce postoperative analgesic comsumption. A meta-analysis with assessment of optimal treatment parameters for postoperative pain. Eur J Pain. 2003; 7: 181-8.

9. Melo de Paula G, Molinero de Paula VR, Dias RO, Mattei K. Estimulação elétrica nervosa transcutânea (TENS) no pós-operatório de cesariana. Rev Bras Fisioter. 2006; 10 : 219-24. dor, visando à humanização do ciclo gravídico-puerperal.

Pode-se concluir a importância deste recurso na humanização do puerpério que visa diminuir as morbidades após cesárea e promover melhor bemestar e conforto para as mulheres realizarem o autocuidado e os cuidados com o recém-nascido. Salienta-se a importância de mais estudos na área, que visem avaliar recursos para o alívio de dor.

\section{Agradecimentos}

Os autores agradecem aos coordenadores e funcionários da Maternidade do Complexo do Aeroporto da cidade de Ribeirão Preto, São Paulo, às puérperas que aceitaram participar da pesquisa $\mathrm{e}$ ao auxílio financeiro Conselho Nacional de Desenvolvimento Científico e Tecnológico (CNPq).

10. Rakel B, Frantz R. Effectiveness of transcutaneous electrical nerve stimulation on postoperative pain with movement. J Pain. 2003; 4: 455-64.

11. Hartrick CT, Kovan JP, Shapiro S. The numeric rating scale for clinical pain measurement: a ratio measure? Pain Pract. 2003; 3: 310-6

12. Castro CES. A formulação linguística da dor: versão brasileira do Questionário McGill de Dor. [dissertação]. São Carlos: Centro de Ciências Biológicas da Universidade Federal de São Carlos; 1999

13. Carrol D, Tramer M, McQuay H, Nye B, Moore A. Randomization is important is studies with pain outcomes: systematic review of transcutaneous electrical nerve stimulation in acute postoperative pain. Br J Anesth. 1996; 77: 798-803.

14. Marques AP, Paccin MS. Pesquisa em fisioterapia: a prática baseada em evidências e modelos de estudo. Fisioter Pesq. 2005; 11: 43-8.

15. Ferreira CHJ, Payno SM. A eletroestimulação nervosa transcutânea como recurso de alívio de dor no trabalho de parto. Femina. 2002; 30: 83-6.

16. Apfelbaum JL, Chen C, Mehta SS, Gan J, Tong J. Postoperative pain experience: results from a national survey suggest postoperative pain continues to be undermaneged. Anesth Analg. 2003; 97: 534-40.

17. Smith CM, Guralnick MS, Gelfand MM, Jeans ME. The effects of transcutaneous electrical nerve stimulation on post-cesarean pain. Pain. 1986; 27: 181-93.

18. Lantsev FA, Smirnov AA. The use of transcutaneous electrical nerve stimulation for postoperative analgesia in parturients undergoing cesarian section. Anesteziol Reanimatol. 1990; 6: 66-9. 
19. Wang B, Tang J, White PF, Naruse R, Sloninsky A, Kariger R, Gold J, Wender RH. Effect of the intensity of trancutaneous acupoint electrical stimulation on the postoperative analgesic requeriment. Anesth Analg. 1997; 85: 406-13.

20. Hoolinger JL. Transcutaneous electrical nerve stimulation after cesarean birth. Phys Ther. 1986; 66: 36-8.

21. Reynolds RA, Gladstone N, Ansari AH. Transcutaneous electrical nerve stimulation for reducing narcotic use after cesarean section. J Reprod Med. 1987; 32: 843-6.

22. Niven CA, Murphy-Black T. Memory for labor pain: a review of the litetarure. Birth. 2000; 27: 244-53.

Recebido em 5 de agpsto de 2008

Versão final apresentada em 22 de dezembro de 2008

Aprovado em 8 de janeiro de 2009
23. Wilder-Smith CH, Hill L, Dyer RA, Torr G, Coetzee E. Postoperative sensitization and pain after cesarean delivery and the effects of single IM doses of tramadol and diclofenac alone and in combination. Anesth Analg. 2003; 97: 526-33.

24. Tsen LC, Thomas J, Segal S, Datta S, Bader AM. Transcutaneous electrical nerve stimulation does not augment epidural labor analgesia. J Clin Anesth. 2001; 13: 571-5. 